Comparative Philosophy Volume 8, No. 1 (2017): 63-81

Open Access / ISSN 2151-6014

www.comparativephilosophy.org

\title{
MALAGASY TIME CONCEPTIONS
}

\author{
CASEY WOODLING
}

\begin{abstract}
In this paper I discuss Øyvind Dahl's argument (1995,1999) for the conclusion that Malagasy people conceive of the future as coming from behind them and not as being before them as most worldviews do. I argue that we have good reason not to attribute this view to Malagasy people. First, it would mark an inefficient and anomalous way of keeping track of the past and future. Second, the linguistic and testimonial evidence presented by Dahl doesn't support the conclusion. Even though this specific argument fails, Dahl has many enlightening things to say about Malagasy time conceptions, such as the various time-conceptions that figure more predominantly in their worldview as opposed to the general modern Western worldview. Dahl is right that successful communication for Westerners in Madagascar requires understanding that the Malagasy worldview is structured more by an event-related conception of time than the general modern Western worldview. I also show in this paper that the three time conceptions Dahl outlines are relevant to living a good life.
\end{abstract}

Keywords: Malagasy, Madagascar, time, time conceptions, conceptual metaphor

\section{INTRODUCTION}

In two works Øyvind Dahl $(1995,1999)$ offers various claims about fundamental time conceptions embedded in the worldview of the people of Madagascar, what I shall call the Malagasy worldview. ${ }^{1}$ Most striking is the claim that Malagasy people see the future as coming from behind them-not as being before them as in most worldviews. According to Dahl, for the Malagasy people it is as if the future comes up from behind them to meet up with them in the present. This is surely distinct from how many worldviews conceive of the relation between individuals, the past, and the future - though there are exceptions. Nunez and Sweetser (2006) argue that speakers of Aymara in the Andes Mountains in South America see the future as being behind them and the past as being before them. Overwhelmingly, though, worldviews

WOODLING, CASEY: Lecturer of Philosophy, Coastal Carolina University, South Carolina, USA. Email: cwoodling@coastal.edu

\footnotetext{
${ }^{1}$ Throughout I use the adjective 'Malagasy' to refer to the things and people of Madagascar.
} 
conceive of the future as being in front of observers and the past as being behind them. ${ }^{2}$ This is certainly true of the modern Western worldview, the general worldview that prevails in industrialized Western countries. Of course, if Dahl were right about the Malagasy worldview, it would be a fascinating discovery. Some of the evidence he provides is linguistic, relying primarily on English translations of Malagasy expressions used to talk about the future, and some of the evidence is based on the testimony of native speakers of Malagasy. ${ }^{3}$ As heady and exciting as Dahl's conclusion may be, it ought not to be believed because none of the evidence he presents supports the claim that Malagasy people conceive of the future as coming from behind them. This is actually a comforting thought, because it is hard to make sense of the idea that the future and the past are both behind observers. ${ }^{4}$ Seeing both the past and future as behind an observer is markedly inefficient when it comes to keeping track of time, as it complicates reference to both the past and future. It is unlikely that such an inefficient way to distinguish the past from the future would have arisen for speakers of a language.

With all that said, Dahl makes some very insightful observations about various conceptions of time at work in the Malagasy worldview, such as conceptually distinct, but often practically overlapping, conceptions of time, which he labels as linear, cyclical, and event-related. He is right that the cyclical and event-related time conceptions are more prominent in the Malagasy worldview than the general modern Western worldview, the latter being structured primarily by the linear conception of time. Dahl is also right that successful communication in Madagascar requires sensitivity to these varying time conceptions and the fact that the event-related conception predominates. I add to these insights at the end of the paper by discussing the interconnections and distinctions between these time conceptions and also how these various conceptions are relevant to living a good life.

\section{THE COHERENCE OF THE IDEA OF THE FUTURE COMING FROM BEHIND}

What does it mean to say that the future is experienced by observers as coming from behind to meet up with them? Here is a description from Dahl (1999).

\footnotetext{
2 In discussing various worldviews, it must be remembered that what is being described are very general conceptual structures. There are obviously many conceptual differences within individuals who share a worldview. And there is clearly overlap between distinct worldviews as well, as I discuss in this paper with regard to conceptions of time.

${ }^{3}$ Malagasy is the language spoken in Madagascar. There are some dialectical variations throughout the island, but these variations do not prevent speakers of distinct dialects from achieving mutual understanding very easily.

${ }^{4}$ Dahl suggests that Malagasy people see the past as present and before their eyes; however, his case for this is unconvincing (see section 3.1 for the reason). So, we are ultimately left struggling to make sense of the idea that both the future and the past are behind observers. I think that the idea can be made coherent (as I explain later), but the problem is that it is a very inefficient way to track time in a language and therefore very unlikely to have arisen.
} 
The observer does not move at all through time-on the contrary it is time that moves from behind and passes the observer. In this way, having eyes only in front of his head, she or he can only "see" present and past events, while the future remains unknown or "unseen". (44)

The idea here is that the future is really behind individuals and it is time that comes up to the present, reaches, and then passes those individuals. To really unpack this idea, it is helpful to consider the work on conceptual metaphor first pioneered by Lakoff and Johnson (1980a and 1980b). They note that some concepts are really metaphorical in that they are understood in terms of other concepts; importantly, we do not realize their metaphorical nature because the metaphors are so basic and common that we take them for granted. There are three types of conceptual metaphors laid out in Lakoff and Johnson (1980a): structural, physical and orientation metaphors. In the case of structural metaphors, one set of concepts is understood and articulated in terms of another. There is the structural conceptual metaphor argument is war. We describe arguments in terms of war. This leads us to think of an objection to an argument as being a counter-attack or as someone who loses an argument as being defeated. We map the characteristics of war onto our characterizations of arguments. Compared to structural metaphors, physical metaphors are more subtle. They involve physicalizing something that is not literally a substance in order to achieve various communicative functions, such as, "referring, quantifying, identifying aspects, identifying causes and setting goals and motivating actions" (Johnson and Lakoff 1980a, 461). For example, "My fear of insects is driving my wife crazy" (ibid). Here an instance of fear is metaphorically treated as a substance to facilitate communication. The fear is physicalized, we might say, in order to refer to it and express its causal connection to the mental states of the wife. Last, there are orientational metaphors, which are crucial for our present purpose because many time conceptions are actually grounded in orientational metaphors. According to Lakoff and Johnson (1980a), this kind of metaphor,

[D]oes not structure one concept in terms of another, but instead organizes a whole system of concepts with respect to one another. We call them "orientational" metaphors because most of them have to do with spatial orientation: up-down, front-back, in-out, onoff, deep-shallow, central-peripheral. These spatial orientations arise from the fact that we have bodies of the sort we have and that they function as they do in our physical environment. Orientational metaphors give a concept a spatial orientation, for example, happy is up. (461)

Time concepts often depend on orientational metaphors. English provides ready examples. English speakers think of the future as being in front of them, as in 'in the weeks ahead of us', and they think of the past as being behind them, as in 'in the weeks behind us'. Lakoff and Johnson (1980a and 1980b) note that time passing is motion is an orientational metaphor that structures our thinking about time. In fact, Lakoff (1993) clarifies this further by saying that there are two species of this 
overarching metaphor: time passing is motion of an object and time passing is motion over a landscape. In the first metaphor, spatial references are fixed to an observer while it is time that moves to the observer. In the second metaphor, it is the observer that moves to meet up with a fixed time. Consider the following expressions from Lakoff (1993) to illustrate the different conceptual metaphors.

Time passing is motion of an object

- The time has come.

- The time for action has arrived.

- Time is flying by.

Time passing is motion over a landscape

- He arrived on time.

- We are coming up on Christmas.

- We're getting close to Christmas.

Both of these conceptual metaphors are species of the more general one that understands time in terms of moment and therefore partially in terms of space. It is useful to not only understand that time is nearly always a metaphorical concept, but that there are distinct conceptual metaphors that can ground thinking about time within a language community. Let us pause to examine how Dahl's thesis relates to these two conceptual metaphors. The easiest way to make sense of the future coming from the past is in terms of the time passing is motion of an object, where it is time that is moving and not the observer. In fact, Dahl (1999) says this much about the Malagasy conception of time: "it is time that moves from behind and passes the observer"(44). Of course, the fact that this conceptual metaphor is operative in Malagasy speakers and hence the Malagasy worldview no more shows that they truly see the future coming from behind than English speakers do, since this very same conceptual metaphor structures the thinking of English speakers. For our purposes, though, it is useful to draw out these conceptual metaphors to better understand the time conception that Dahl attributes to Malagasy people.

One immediate difficulty with the attributed time conception is the challenge of distinguishing the past from the future. ${ }^{5}$ If the past and the future are both behind an observer, then it is not clear how to distinguish them. The main reason that orientational metaphors arose is that they help speakers to efficiently organize their talk and thought about time. If a worldview metaphorically locates both the past and future behind an observer, it seems to make the orientational metaphor very

\footnotetext{
${ }^{5}$ Notice that the suggestion that Aymara speakers see the future as behind them and the past as in front of them doesn't have this difficulty. Nunez and Sweetser (2006) argue for this claim, and they make a crucial appeal to gestures made by Aymara speakers as well. I do not discuss their case here, but it is worth noting that the Aymara inversion of the normal front/future and back/past mappings would surely be anomalous with regard to other languages and worldviews - but not inefficient because it would not map the future and past to the same spatial location.
} 
inefficient. It is true that one could still distinguish the concepts past and future on such a worldview by using the following concepts the time behind an observer that has already been experienced and the time behind an observer that has yet to be experienced. However, it would be an unnecessary complication and an oddity for sure. We should avoid attributing such conceptions, because it would mark an anomalous instance where a worldview is not structured by an efficient conceptual metaphor that understands time in terms of space and motion in a way that improves the efficiency of communication.

\section{THE EVIDENCE FOR THE VIEW}

All of that is to say that we have very good reason to scrutinize the evidence Dahl provides for the time conception attributed to Malagasy people. Dahl claims that there is linguistic evidence for the attribution, and also that Malagasy people admit that this is how they conceive of time. I do not question the type of evidence presented, because I think that this type of evidence could theoretically support a conclusion about some aspect of a culture's worldview. My objection is that the specific evidence provided doesn't support the conclusion that Malagasy people see the past as coming from behind. Let me discuss the linguistic evidence first and the testimonial evidence second.

\subsection{THE LINGUISTIC EVIDENCE}

Dahl's case relies in part on translating various Malagasy terms and phrases used to talk about the future. Here is a prime example. The term 'aoriana' is used to talk about the future, for example, 'Hovitako ny asa aorian' ity herinandro ity', which means The job will be finished by me after this week. 'Aoriana' also means behind, as seen in a popular proverb cited by Dahl: 'Manao dian-tana: banjinina ny aloha, todihina ny aoriana', which means Do as the chameleon when walking: look forward and turn the other eye backward. The polysemy of 'aoriana' is a key part of Dahl's case: 'aoriana' is often used as an adverb of time to talk about the future while it is also used as a preposition that means behind. He contrasts the idea that the future comes from behind with Malagasy talk about the past, which he says suggests that Malagasy people see the past as closely linked to the present. He notes that the Malagasy phrases 'teo alohan'ny maso' and 'taloha' are used to talk about the past, and holds that both convey the sense that the past is right before one's eyes as 'alohan'ny maso' means before the eyes. Of course, students of Malagasy know (as does Dahl) that ' $t$ ' is used as a tense marker to indicate that the past is being talked about, so the most accurate translation of 'teo alohan'ny maso' is not-as Dahl claims - there before one's eyes but there before one's eyes (in the past). So, it's not as if these Malagasy ways of talking about the past provide evidence for thinking that the past is somehow contemporaneous with the present for them or that it is as in Aymara where the past is seen as in front of one, because this Malagasy talk about the past translates to mean something like what was before one's eyes. Furthermore, the 
evidence that comes from the use of 'aoriana' doesn't support the idea that the future comes from behind either. Consider a possible etymology of the term. 'Aoriana' was first a preposition that simply meant behind and then got new life as an adverb of time because the future being behind something in an observer's environment is an apt metaphor to talk about the future; it is natural after all to think of future time as being metaphorically hidden from the view of observers as if it were behind something in the distance located physically in front of one, such as a mountain, for example. ${ }^{6}$ The etymology just sketched is a more plausible explanation of how 'aoriana' came to be used to talk about the future than the strange idea that it was used to talk about the future because observers understood the future to be behind them. In short, because the etymology I suggest is plausible, and it doesn't attribute an inefficient way of tracking time to the Malagasy, it ought to be preferred.

While it is primarily the polysemy of 'aoriana' that drives Dahl's case, he presents other evidence as well. He notes, for example, that the phrase 'amin'ny manaraka' is used to talk about the future, which is certainly correct. Malagasy people often end conversations with 'amin'ny manaraka indray', which means see you next time. It is also common to use 'manaraka' in the following way. 'Handeha any Antsirabe aho amin'ny herinandro manaraka', which means I am going to Antsirabe next week. Dahl notes that 'manaraka' can also mean behind in addition to being an adverb of time that tracks the future just like 'aoriana'. The problem here, though, is that there is no such sense. 'Manaraka' is an active verb that means (variously) to follow; to obey; or to prosecute and is also used as an adjective meaning next or following. ${ }^{7}$ It also is used, as I noted, in the expression 'amin'ny manaraka indray'. However, there is no reason for thinking that it has a special sense meaning behind as 'aoriana' does. Of course, 'to follow' can connote the idea that one thing is perhaps behind another, as in 'The students followed the teacher in line'. However, 'manaraka' doesn't have this locational sense. The notion of 'to follow' that is best expressed by 'manaraka' is the sense expressed by 'to follow an example,' as in 'manaraka efa' which means to do as others do. So, 'manaraka' is not used to mean to follow behind another.

Additionally, Dahl presents other evidence related to the following adverbs of time: 'ho avy' and 'amin'ny ho avy'. These phrases mean the time that will arrive. 'Avy' is also used to mean from, as in the common question 'Avy aiza ianao?' which means Where are you from? or Where did you come from? Dahl (1999) says that the use of 'ho avy' "reinforces the impression of time as a moving phenomenon with respect to the observer" (44). In other words, it is evidence of the conceptual metaphor time passing is motion of an object at work in the Malagasy worldview. This point is fair enough but the use of 'ho avy' to talk about the future hardly supports the idea that Malagasy people see the future as coming from behind. After

\footnotetext{
${ }^{6}$ In fact, native speakers have confirmed just this. Consider the sentence, 'Hihaona aminao aho aorian'ny Krisimasy', which means I will meet with you after Christmas. Native speakers have confirmed that this is a way of saying that the time of the appointment, the time of the meeting, is hidden by Christmas, in the very same way I sketch in the possible etymology above. I thank Graziella Masindrazana for help with this point.

${ }^{7}$ Malagasy has three voices for verbs: active, passive and circumstantial.
} 
all, people in English say that the time for $X$ has not yet come or that that time is catching up to one, and thus think of the future by way of the conceptual metaphor time passing is motion of an object. And no one would attribute to English speakers the idea that the future comes from behind. In short, the linguistic evidence involving adverbs of time does not support the time conception that Dahl attributes to Malagasy people, and nothing from the discussion of conceptual metaphor does either.

I should also note here that linguistic evidence similar to that presented by Dahl was presented to me by Professor Jean de la Croix Malazamanana of the University of Antananarivo. Typically, 'ambony' and 'ambany,' which mean up and down, respectively, are used not only to talk about physical location but also time. ${ }^{8}$ For example, someone might say, 'Ho any Antananarivo aho amin'ny herinandro ambony', which means I will be in Antananarivo next week. So, 'ambony' is used to mean something like next or forthcoming, while 'ambany' is used to mean last or past when they function as adverbs of time. This appeals to a conceptual metaphor the future is up and the past is down. Professor Malazamanana reported that some people invert this usage and use 'ambony' to talk about the past and 'ambany' to talk about the future, suggesting that some people not only think about the future as being down and therefore perhaps behind them, but also think about the past as being up and therefore perhaps before them. This reversal of the typical front/future and behind/past mapping would seem to lend support to Dahl's conclusion. However, Professor Malazamanana also noted that this usage could track the physical layout of calendars, where past weeks are listed above more current or future weeks. When one mentally pictures a calendar, it is natural to think of one week as being above (being up) or below another (being down). Perhaps that is what explains the inverted usage of 'ambony' and 'ambany'. Again, as before, we have an explanation of usage that doesn't attribute an anomalous worldview where past and future are both referred to using the same location, and thus the proffered etymology should be preferred. The lesson here in fact is the same as the case of 'aoriana'. We must be wary of conclusions about radical features of conceptual frameworks that are supported merely by points involving translation. It's true that 'aoriana' is an adverb of time that tracks the future and a locative preposition that means behind. From this, we cannot conclude that Malagasy people see the future coming from behind. That is much too quick. The same must be said of the cases that Professor Malazamanana reports. The inverted usage of 'ambony' and 'ambany' could be tracking not a radical feature of worldview but the mere fact that people are thinking of time, on some occasion, in terms of the spatial layout of a calendar, where past weeks are above future weeks (or up) and future weeks are below (or down).

Dahl also uses common expressions and greetings as evidence for his conclusion. When the new year comes, Malagasy people great each other with some version of 'tratry ny taom-baovao', which means Happy New Year, but which would be literally

\footnotetext{
${ }^{8}$ Notice that this would involve the time passing is motion over a landscape metaphor, which is further evidence that worldviews often contain various conflicting conceptual metaphors that structure thinking about time. Malagasy and English are two examples.
} 
translated by most as the New Year is caught (or attained), as 'tratra' means to be caught or to be attained. Of this expression, Dahl (1999) says, "It is not the person who reaches the year, but the year that catches up with the person. The observer is stationary, and time is a moving phenomenon, arriving from behind with respect to the observer" (44). This conclusion is not warranted at all, however, given what Dahl says. It is entirely possible to read the expression in a way that the New Year is attained or caught by the person and not in a way that the year catches up to the person from behind. ${ }^{9}$ In the past, the expression was 'Arahabaina, nahatratra ny taona ianao', which means Congratulations, you have made it to the new year. ${ }^{10}$ 'Mahatratra' literally translates as caused to be caught or attained as 'maha' is a prefix that indicates causation. Even going back to the older expression, there is still not enough evidence to support Dahl's conclusion. It's entirely possible to read the expression as meaning the same thing as: 'Congratulations, you made it to the New Year.' In fact, 'tratra' sometimes means the same thing as 'tonga', which means to come or to arrive. The traditional way to say 'welcome' in Malagasy is 'tonga soa'. However, it has been reported that 'tratra soa' is now common as well, drawing our attention back to the two possible senses of 'tratra' noted earlier. It can mean caught or it can mean attained. So, it must be admitted that the original sense of 'tratra' in 'tratry ny taona' is not entirely plain. ${ }^{11}$ It could be either the sense meaning to be caught or the sense meaning to be attained. Even if it did become clear that it was the sense that means caught, that is still not enough to show that Malagasy people see the future as coming from behind, because the meaning would just be: You have caught up with the New Year. If the original expression was 'Nahatratra anao ny taombaovao', which means The New Year has caught up to you, then that may perhaps lend some support to Dahl's conclusion, but the original expression, as I noted, was 'Nahatratra ny taona ianao', which means You have caught up with the New Year, so there is nothing in the use of this very common expression that supports the idea that the Malagasy see the future as coming from behind to meet up with them. ${ }^{12}$ All this shows is that at times the conceptual metaphor time passing is motion over a landscape is at work in Malagasy speaking and thinking about time.

\footnotetext{
${ }^{9}$ In other words, the expression as it is currently used is ambiguous between the two conceptual metaphors of time we have been discussing.

${ }^{10}$ I thank Professor Graziella Masindrazana for drawing my attention to the etymology of this expression. Given this etymology, we can see that the time passing is motion over a landscape metaphor is operative.

11 'Tratra' is transformed into 'tratry' in the famous expression due to rules about permutation for verbs ending in '-tra'.

12 Dahl notes that there are other metaphorical ways of talking about time in Malagasy such as 'miandry fotoana', which means killing time; 'mihazakazaka ny fotoana', which means time flies; and 'mangataka andro', which means to stall. However, he draws no conclusions about the worldview from these expressions but merely notes them in passing. In this regard, Malagasy is a language like any other in that there are metaphorical expressions used to talk about time.
} 


\subsection{THE TESTIMONIAL EVIDENCE}

In addition to citing evidence based on translation, Dahl cites testimonial evidence from conversations with native speakers of Malagasy. Here is a key passage.

When Malagasy informants were asked about time orientation, they sometimes became confused and did not always agree as to whether the future was in front or behind. Further inquires indicated that they usually did not consciously think about time in oriented categories in their day-to-day affairs. Nonetheless, they always agreed that the metaphorical expressions "before," "behind," and "above" had the designated locative meanings, and that they days and the years caught up with us, not the opposite. (44)

Before we interpret this data, and unpack the quote, it is good to remember that even among native speakers conversations with metalinguistic elements often quickly become confusing. In this case, we have Dahl, a fluent, but non-native speaker of Malagasy, engaging native speakers in discussions that require a high level of metalinguistic awareness for success. No doubt Dahl, since he is driving the inquiry, has the proper level of metalinguistic awareness; one wonders, though, whether the interlocutors also have the right level of metalinguistic awareness. I can speak from first hand experience that many times when a conversation with a native Malagasy speaker moves to talking about the Malagasy language itself, native speakers often become confused. Of course, this is not a feature of just Malagasy native speakers, but is a more general feature of discussions that require metalinguistic perspective. So, it should be no surprise that Dahl's interlocutors often became confused in discussions about whether the future comes from behind, as these conversations had metalinguistic turns. Having said that, another key point is that Dahl says that Malagasy people "did not consciously think about time in oriented categories in their day-to-day affairs", which completely undercuts his claim that they see the future as coming from behind. To understand the future as coming from behind is to think of time in an oriented category, so it's as if Dahl is saying that Malagasy do and do not think of time in oriented categories which is clearly a contradiction. Finally, Dahl says that Malagasy agree that the ways of talking about time had their designated locative meanings. The simplest way to understand this is to understand that Malagasy people confirmed that some adverbs of time are polysemous and can function as locative prepositions as well. So all this says is that, for example, 'aoriana' is used, respectively, to track the future and to track location. The only part of the above quote that cuts any ice with respect to Dahl's thesis about the future coming from behind is the last claim: that Malagasy agree that the days catch up to one and not the other way around. Of course, we need to hear much more here to be convinced of Dahl's main thesis. To what exactly did the native speakers assent? We need to hear about how the questions were posed, and how the responses were stated. If one is asked whether the years caught up with one or if one caught up with the years, then they have been presented with a false dichotomy. There are other options. One might think that both ways of talking about time and observers are somehow 
misleading. So, we cannot simply take Dahl's word here about the content of the testimony. We need to know what questions were asked and what answers were given to properly evaluate this testimonial evidence in more detail. And that evidence is lacking.

\section{THREE WAYS OF TRACKING TIME AND INTERCULTURAL COMMUNICATION}

So far I have been critical of Dahl's discussion of Malagasy time conceptions. I have argued that none of the evidence he presents supports the idea that Malagasy see the future as coming from behind them. It would do a serious disservice to his work on the Malagasy worldview to stop here, however, as he has very insightful things to say about Malagasy time conceptions. Dahl discusses three generic but distinct ways of tracking time that, to various degrees, structure the Malagasy worldview, and other worldviews as well: linear, cyclical, and event-related conceptions of time. ${ }^{13}$ Let me first distinguish these views with the help of Dahl's work. To do this, it is helpful to draw attention to two dimensions on which conceptions of time can be measured.

\section{- Abstractness/concreteness \\ ○ Topology (linearity/circularity)}

First, let us discuss the dimension of abstractness/concreteness. Various conceptions of time can be measured in terms of the degree to which they can abstract away from the concrete world to measure time. The linear, cyclical and event-related conceptions all vary along this dimension. Time conceptions that are graded as more abstract than concrete are marked by greater employment of units of time that are not tied to anything concrete. For example, seconds, minutes, and hours are all units of time that are not directly tied to anything concrete in the physical world. On the other hand, units or markers of time such as the sunrise, the sunset, when the work is done, and when he arrives are clearly tied to more concrete phenomenon. Time conceptions where time is more frequently measured in terms of concrete markers or units will be graded as more concrete than views that employ less concrete markers or units. ${ }^{14}$

The next dimension is the topology or shape of time. Time conceptions can also profitably be graded in terms of the metaphorical shape that the conception grants to time. More linear conceptions of time see time as a linear phenomenon that moves forward into the future as opposed to a circular phenomenon where some previous time is revisited. Friedrich Nietzsche's doctrine of eternal recurrence is a very clear, albeit extreme, example of the cyclical conception of time. ${ }^{15}$ Time literally repeats

\footnotetext{
${ }^{13}$ As I noted earlier in actual worldviews these time conceptions often overlap.

${ }^{14}$ Implicit in this way of ranking views is the idea that these are not binary categories where a view is either abstract or concrete full stop. Views can be more abstract than others or more concrete than others.

${ }^{15}$ Whether Nietzsche meant this view to be a mere thought experiment or a serious description of reality is not to the point here.
} 
itself in the sense that the events of one's life happen over and over again. Less extreme versions of cyclical conceptions of time involve seeing events as happening in a very similar way to what happened before. Dahl notes that such a conception of time is typically found in very agrarian societies where people structure the time of their lives around the seasons, which of course are very similar over time.

There is no direct correlation between the two dimensions sketched above. For example, there is no reason that more abstract theories must be linear or why more concrete theories must be cyclical. These are merely two prominent dimensionsthough not the only dimensions - on which to measure different conceptions of time. Having sketched these two dimensions, let us turn our attention to the three distinction conceptions of time outlined by Dahl. It is important to bear in mind two things, first, that it is possible to see these conceptions melding and overlapping in specific worldviews, and, second, that no worldview's conception of time fits exactly one of these conceptions. It is best to think of worldviews featuring one of these conceptions prominently while also containing the others as well.

The first time conception that Dahl describes is the linear conception of time. This view sees time abstractly and as moving forward in a straight line. This is the way that time is conceived of in the modern Western worldview. ${ }^{16}$ As its name indicates, this conception of time does not view time as cyclical and reoccurring. Time stretches out forward into the future, but it does not repeat itself. Once a certain time passes, it is past and it is not returned to. Additionally, people in the modern Western world and other developed countries think of time in terms of units of measurement. Time is something one has and it can certainly be used up. Time is tracked more frequentlythough not exclusively - by abstract units of time measurement, such as seconds, minutes, days, weeks, and so on.

Such a conception of time has its virtues. It can be used by people to successfully coordinate communication and action even when these individuals do not share the same concrete frame of reference, such as living in the same physical space. In this sense, the abstractness and regimentation of time into segments inherent in this conception of time gives it more universalizability than the other conceptions of time. ${ }^{17}$ In environments when this conception predominates among people, meetings happen at preset times and they can be fixed well into the future. Linear conceptions are integral to detailed planning about the future. Here is a description from Dahl (1999).

This linear concept can be manipulated without reference to concrete events; it is abstracted as an objective standard constituted of a homogenous flux of durations. It is measurable in equal units and free from any concrete reality. Whether or not things happen, this quantitative time passes from a distant past and toward a coming future,

\footnotetext{
${ }^{16}$ Derek Parfit (1984) offered a striking image for such a conception, though his main point is about the identity of the self over time: "My life seemed like a glass tunnel, though which I was moving faster every year, and at the end of which there was darkness" (281).

${ }^{17}$ I thank an anonymous reviewer for helping me see the connection between the abstractness and universality here.
} 
which through the introduction of this abstract time concept becomes predicable and tangible. (46)

Of course, there may be drawbacks to this way of conceiving of time, as it can no doubt be a source of psychological stress when one is constantly counting time and worried about wasting it.

In contrast to linear conceptions of time, cyclical conceptions see time as repeating. This conception of time is at work in agrarian societies or in other societies where people live closely with the earth-both societies of the present day and of the past. Of this conception, Dahl (1999) writes,

The passage of solar and lunar sequences has probably led to the perception of time as cyclical. To see human lives following one another or reconstituting one another in the sequence of generations further inspires a cyclical model. The rhythm of the lives of people and animals during the season of the year promotes the idea that time consists of ever-repeated cycles revolving in an endless rhythm. (47)

What Dahl describes here is not Nietzsche's version of eternal recurrence but rather a way of seeing the passage of time as involving regular pattern repetitions. When one's activities are more concretely tied to the seasonal changes of one's environment, to the land and to animals, then the cyclical model is more likely to predominate. In the end, some versions of the cyclical model are compatible with a linear conception of time. It is possible to have one's thinking about time largely driven by repeatable patterns and also to see time as moving into the future. Dahl (1999) notes that there is no contradiction between versions of the cyclical concept and the linear concept. ${ }^{18}$

Consecutive cycles, however, are not necessarily identical. Cycles can be thought of as a spiral where each cycle has moved a little compared with a previous one. Thus a cyclical metaphor does not contradict an evolution across time, nor is it contrary to a linear time metaphor, since parts of the cycles can also be studied as linear movement. (48)

So, what distinguishes the linear and cyclical conceptions of time? One key difference is that time is seen as less of a resource on the cyclical conception. Because regular patterns of life continuously reoccur, there is much less of a worry that time is getting wasted when compared to a linear conception of time, where the focus on units of time tends to cause individuals to worry that these units are being used up. Lakoff and Johnson (1980a and 1980b) note that the conceptual metaphor time is money is operative in the modern Western worldview. Here is a description.

Time in our culture is a valuable commodity. It is a limited resource that we use to accomplish our goals. Because of the way the concept of work has developed in modern Western culture, where work is typically associated with the time it takes and time is

${ }^{18}$ It should be noted, though, that the doctrine of eternal recurrence does conflict with a linear conception of time. 
precisely quantified, it has become customary to pay people by the hour, week, or year. In our culture time is money in many ways: telephone message units, hourly wages, hotel room rates, yearly budgets, interest on loans, and paying your debt to society by "serving time." These practices are relatively new in the history of the human race, and by no means do they exist in all cultures. They have arisen in modern industrialized societies and structure our basic everyday activities in a profound way. $(1980 \mathrm{~b}, 8)$

Cyclical conceptions of time are not structured by this conceptual metaphor. This is the biggest difference between linear and cyclical conceptions of time. If we will more or less come back to a previous time again - for example, winter will come again - then there is not the same level of worry that time is a resource that is lost if not used or that something precious is being used up. On the cyclical conception, time is not seen as having the same as features as money. So, as a consequence of lacking this conceptual metaphor, this way of thinking about time will bring less stress to those who use it, although there will likely be less accomplished given the more relaxed attitude about time.

Last, there is the event-related conception of time. This conception of time features prominently in the general Malagasy worldview, along with the cyclical view in rural settings. The event-related conception of time involves very concrete tracking of time; there is not a great focus on measuring and tracking time in terms of abstract units. ${ }^{19}$ The main distinction between this conception and the linear conception is that events and not abstract units of measurement are the key drivers in thinking about time. Dahl (1999) explains as follows:

Every action and one's relations with others are organized from the experience of an event. When something happens, for instance, when the roof starts leaking-one takes action, but not before, even if one is aware of the damage to the roof. The event- the leak - triggers the action - the repair. It is not a result of conscious planning, but, rather, a result of a happening. (48)

Dahl offers illuminating anecdotes to illustrate how this conception of time is at work in Madagascar. Those who have ridden a bush taxi (the buses that travel in the bush between larger cities) in Madagascar will have experienced the event-related conception first hand, for they know that these vehicles leave not at present times but when the event of them being full occurs. ${ }^{20}$ On a modern Western conception,

\footnotetext{
${ }^{19}$ This is true even though time is tracked using units of measurement in worldviews, such as the Malagasy worldview, where the event-related conception predominates.

${ }^{20}$ I cannot forebear here and must mention a fond memory I have of learning of this event-related conception first hand. I was an American Peace Corps Volunteer in village named Ambatofinandrahana from 2007 to 2009. I taught English at the local high school. A new semester was approaching, so my counterpart and I were discussing the date of the regular teacher meeting. I was perplexed as he explained that the meeting would happen when it was time. "Well, what day is that?" Once I learned the date, I would of course ask about the hour that the meeting would start. I got no such answer regarding the date, however, so there was no way to ask my anticipated follow-up question about an even more specific time. My counterpart endeavored-very patiently-to explain that the meeting would happen when it was time-when the time was ripe. It would not happen before
} 
departures happen at preset times, but not so in most transportation situations in Madagascar. So, on the dimension of abstractness/concreteness, this conception of time is the most concrete of the three discussed. The practice of famadihana (exhumation) in Madagascar has been well documented. It is especially prevalent in the central highlands. In addition to celebrating with food and drink, families remove corpses of deceased relatives from their tombs, remove the cloth wrapping them, and wrap them in fresh cloth in order to honor and celebrate the life of their ancestors. These events happen not according to some formula grounded in the linear conception, such as exhume the ancestors every 7 years. They happen when the ancestors come to family members in dreams. It is only after the event of this dream visitation that the family knows it is time to conduct the exhumation and celebration.

The event-related conception by itself entails neither the linear nor cyclical conception, which is to say that there is nothing of the linear or cyclical conceptions built into the event-related conception. It is a feature of the event-related conception that not much planning is done for the future, as planning for the future requires one to imagine various possible future situations, which are not concrete events. The event-related conception of time, Dahl notes, typically melds with a cyclical conception in the Malagasy countryside. It is probably not uncommon to see these two conceptions at work in one overall conception of time, as they are tied more to concrete changes in the physical environment of observers. It should be noted that a strict event-related conception of time would be even less forward looking than a cyclical conception of time. On a cyclical conception, one can plan into the future as one sees the various patterns of time as repeating. An extreme version of the eventrelated conception would be one in which very little planning for the future is done as all one's thinking about time is tied to events that occur and not events that could occur. Such an extreme version is not found in normal adult human beings, however, but is likely found only in very young children and perhaps some animals.

Of course, no worldview is structured rigidly by just one of these conceptions. All three are at work in many actual worldviews. Dahl brings this out nicely when he notes that in social settings, such as parties, the event-related conception of time becomes prominent in the modern Western worldview. However, it is profitable to note for cross-cultural understanding that one of these conceptions is more prominent in a given worldview. In the general Malagasy worldview, the event-related conception of time is so prominent that it has given rise to a very common expression 'fotoan-gasy', which literally means Malagasy time. People will often use this expression, especially around foreigners, to express different norms of time in modern Western and Malagasy cultures. Time is flexible in many social settings in Madagascar. Things do not typically happen at preset times, for daily planning is not set by abstract units of time - at least certainly not to the extent that it is in modern Western societies. There is more improvisation when it comes to meetings and other social events, given that one must often wait for certain events to trigger meetings. If

then or at a set time. It would happen when certain events fell into place, such as certain teachers returning to town from their holiday. 
an event occurs that prevents someone from coming to a meeting, there is no problem whatsoever given that person's lateness. In a Western conception, outside of a special class of emergencies, individuals would be held accountable for being late as it is believed that they should plan for contingencies that cause such lateness.

Dahl rightly notes that in any individual - and in any general worldview for that matter-you have a melding of these three conceptions. And he is surely right that successful living and communication in Madagascar require that one realize that an event-related conception of time is a central part of the Malagasy worldview and culture. To effectively communicate with Malagasy people one must realize that the event-related conception structures many Malagasy people's thinking about time. Of course, there are elements of linear thinking as well, but if one has the expectation that events will happen at preset times, then disappointment is inevitable. Dahl (1999) offers an apt summary.

When the urban Westerner acts and makes plans according to a linear time concept and the Malagasy acts according to an event-related time concept, they are likely to misinterpret each other's actions, and communication will become problematic. In the cases narrated above, the Westerner will probably judge the Malagasy partner in terms of stereotypes of being inefficient, undisciplined, and irresponsible, and showing little interest for benefits and lack of productivity. (56)

One must understand key aspects of an interlocutor's worldview for successful communication to be achieved. That is a non-negotiable truism. It is essential for Westerners to understand that Malagasy thinking about time is more driven by events than abstract signposts that measure time. There is simply a more relaxed attitude about time built into the Malagasy worldview. It is, as Dahl reminds us, as ingrained in Malagasy as the linear conception is ingrained in Westerners. Unless one has a great amount of detail about a Malagasy interlocutor's worldview, then it must be assumed that they are working with the event-related conception of time, not to the exclusion of the other two conceptions, but it is likely that they have internalized the event-related conception to a great extent.

We can now ask the following philosophical questions about the three conceptions of time:

(1) To what extent are these time conceptions compatible in a given worldview, that is, what is their logical relation?

(2) Are there any benefits in terms of a good life to one of these conceptions of time?

I have already answered (1) to some extent by reinforcing Dahl's idea that in any actual worldview, versions of these various time conceptions are compatible, with one or more of the conceptions being at the fore of any actual worldview. For example, in the modern Western worldview, the linear conception is most prominent and in the Malagasy worldview the event-related conception is most prominent. One 
would find some incompatibilities in extreme, purely theoretical versions of these time conceptions. For example, the doctrine of eternal recurrence sketched by Nietzsche is not compatible with the linear conception of time, because the linear conception of time sees time as being used up and not repeating, where Nietzsche's famous conception sees time as recurring exactly as it has before. A strict eventrelated conception of time is not compatible with either a cyclical or linear conception of time, because an exclusive focus on concrete events prevents planning for the future which requires contemplating possible events. In actual practice, though, the three time conceptions are all elements, to various degrees of prominence, in any actual worldview. So, the answer to (1) is that only in their extreme forms are these time conceptions mutually exclusive. In practice, they meld together and overlap in all actual worldviews.

I think that Malagasy time conceptions can teach us lessons about the good life; a conscious appreciation of the time conceptions discussed above can contribute to a good life. ${ }^{21}$ If one does not reflect on one's own thinking about time, and one falls into thinking exclusively with one of these conceptions of time, then this lack of reflection can detract from a good life. The key point about time and a good life is seeing that the various time conceptions have their appropriate application at certain times in a life.

In environments that require social cooperation to achieve a mutually desired result, the linear conception of time is most appropriate, given its abstractness and therefore its ability to allow people who may not share the same physical reference points to better coordinate their collective action. This is the conception of time that best facilitates personal accomplishment as well given that it allows for efficient planning and structuring of projects. And this is likely the conception of time that is most advantageous to the acquisition of knowledge as well given that acquiring knowledge requires discipline, planning and often coordinating efficiently with others. This is not the conception of time that necessarily enhances personal relationships the most, but it does allow for a level of mutual achievement not achievable with the other conceptions. Despite these benefits, using this conception of time exclusively is not conducive to living a good life, as it increases stress and gives one the sense that one must always be doing something because time is valuable and it is continually being used up. There is great stress is trying to fill every minute of the day with productivity. This is the main drawback of full-time use of this time conception.

\footnotetext{
${ }^{21}$ I assume that some objective list of necessary and sufficient conditions is what constitutes a good life. Not all philosophers agree that this is the correct way to characterize a good life, instead trying to reduce the good life to one factor such as desire-satisfaction (desire theories) or pleasure or happiness (hedonism). I cannot sort out all these issues here or offer an argument for objective list theories. I merely show how these ways of thinking about time are relevant to the good life on the plausible view that a life is measured as good based on how well is satisfies an objective list of features, such as leisure and relaxation, productivity and accomplishment, friendship, knowledge, familial love, romantic love, physical love, bodily health and autonomy, and proper perspective between self and the universe, among other plausible features.
} 
Like the linear conception, the event-related conception has real virtues. It allows for more relaxation, improvisation, and creates less stress for an individual. It allows one to disconnect from one's larger projects and achieve true leisure. It also opens up space for more improvisation and new discoveries. Adopting this time conception facilitates leisure for a Westerner, as leisure must be divorced from work, and the linear conception of time is tied up with thinking about work in the modern Western worldview.

Another benefit that must be mentioned is that this conception of time can actually, at times, facilitate achievement and success in personal and interpersonal endeavors - a virtue previously attributed to the linear conception of time. It is indeed important to organize our lives according to preset times, which does require the linear conception; however, we need to remain sensitive to when it is most appropriate for activities to happen. The focus on whether an event is ripe for happening, which one sees in the event-related conception, is valuable for action. For instance, if a key strategic partner cannot make a meeting, then it should be cancelled even if a time for the meeting has already been set, because without the key partner the meeting will not be productive. The meeting is not ripe for happening so another time should be sought. It turns out that the event-related conception of time is helpful to keep in mind for successful planning, though planning should be largely driven by the linear conception of time.

If the event-related conception is used exclusively, however, it detracts from a good life, as it is not a conception of time which best facilitates personal and interpersonal achievement, as achievement undoubtedly requires planning, and this is not a conception of time that facilitates planning (although it is helpful to bear in mind as it helps to track the ripeness of events). Provided that relaxation and leisure, personal and interpersonal achievement, and knowledge acquisition are all part of a good life, then both of the event-related and linear time conceptions are crucial ingredients to the reflection and thinking necessary for living a good life.

What about the cyclical conception? The cyclical conception is important for providing the proper understanding of the relationship between self and universe. Seeing that patterns repeat, that there is a sense in which various events will occur in similar ways to what has past, and that events will continue to occur in similar ways even after one's life is over all help to give individuals proper perspective. We can see that our own lives and overarching projects are not of immense significance when compared with the past and future of the entire universe. This is the sort of phenomenon discussed by Thomas Nagel in various places. In "The Absurd", he offers the following.

Each of us lives his own life-lives with himself twenty-four hours a day. What else is he supposed to do-live someone else's life? Yet humans have the special capacity to step back and survey themselves, and the lives to which they are committed, with that detached amazement which comes from watching an ant struggle up a heap of sand. Without developing the illusion that they are able to escape from their highly specific and idiosyncratic position, they can view it sub specie aeternitatis - and the view is at once sobering and comical. (720) 
Although Nagel is offering an explanation for the absurdity of life, the cyclical conception of time enables us to take up this detached perspective that Nagel famously discusses here and other places. ${ }^{22}$ Seeing our proper place allows us to avoid the trap of seeing one's overarching projects as all-important and therefore is a good hedge against them becoming all-consuming. Conceiving time with the linear conception can lead to projects being felt as all-consuming, which brings too much stress. Periodically taking on the detached, cosmic perspective Nagel talks about provides a healthy perspective that is necessary for a good life. Of course, one ought not to inhabit the cyclical conception of time all the time, as it would surely detract from personal and interpersonal achievement, as there are times when one must focus narrow-mindedly on an individual or collective project, and while the cyclical conception of time does allow for planning for the future, there is less urgency to our actions given that there is a real sense in which there will always be more time in the future to achieve what is needed.

In the end, it seems that the proper perspective on time and a good life requires individual reflection and a conscious attempt to embody these various conceptions of time at different times in a life. Thinking with just one of these conceptions would not allow one to live a life with all the features of a good life sketched so far: personal and interpersonal achievement, knowledge acquisition, leisure, relaxation, and the proper perspective between one's life and the immensity of the universe. What allows us to gain this important insight is a look at Malagasy time conceptions. Understanding the event-related conception helps us to gain perspective on our own thinking about time and the tacit commitment in the modern Western worldview to a linear conception of time. This is one of the key lessons to learn from the Malagasy worldview: that the event-related conception of time has a role to play in allowing us to achieve the proper leisure and relaxation necessary for a good life while it also helps us with planning in so far as the ripeness of event is important.

\section{CONCLUSION}

In this paper I have discussed Malagasy time conceptions, especially Dahl's idea that Malagasy people see the future as coming from behind. I argued that we have good reason not to attribute this conception to Malagasy people, as it would mark a very inefficient and problematic way of distinguishing the past from the future. I also argue that there is no good reason to attribute this conception on the basis of the evidence provided by Dahl. To leave things there, though, would be a great disservice to Dahl's work on communication in Madagascar and the Malagasy worldview, because he has incredibly insightful things to say about many aspects of the Malagasy worldview. I have mentioned but a few of these insights, the ones related to time. Serious students of the Malagasy worldview would benefit greatly from reading for themselves many more insightful details in Dahl's work. In addition to the point that

${ }^{22}$ Perhaps most famously and thoroughly discussed in Nagel (1986). 
sensitivity to the various time conceptions that Dahl sketches have practical implications when it comes to communication with Malagasy people, there are interesting philosophical lessons that should be drawn from the three conceptions of time. In this paper, I have sketched out their logical relations, showing that in actual worldviews these three conceptions meld, and are more or less prominent depending on the worldview. In their extreme versions, they are mutually incompatible, but these extreme versions exist in theory only and are heuristic devices for describing the actual features of time conceptions in existing worldviews. There are also important points to see about thinking about time and a good life, especially lessons to draw about Malagasy thinking about time. I list some necessary and sufficient features for a good life in the paper, and argue that to achieve them one must make a conscious effort to embody the three different time conceptions at the appropriate time in one's life.

\section{ACKNOWLEDGEMENTS}

I thank Graziella Masindrazana, Hobiniaina Rabarivelo, and Jean de la Croix Malazamanana for helpful discussions of this topic. My thanks as well to an anonymous referee who provided very useful comments. In addition, I thank the U.S. Fulbright Program, which helped to support this research while I was a Fulbright Scholar at the University of Antananarivo (2016-2017).

\section{REFERENCES}

Dahl, Øyvind. (1995), "When The Future Comes from Behind: Malagasy and Other Time Concepts and Some Consequences for Communication", International Journal of Intercultural Relations 19: 197-209.

- (1999), Meanings in Madagascar: cases of intercultural communication (Westport, CT: Bergen and Garvey).

Lakoff, George (1993), "The contemporary theory of metaphor", in Metaphor and thought, edited by Andrew Ortony (Cambridge: Cambridge University Press), 202-51.

Lakoff, George and Johnson, Mark (1980a), "The Metaphors in Everyday Language", Journal of Philosophy 77: 453-486.

- (1980b), The Metaphors We Live By (Chicago, IL: University of Chicago Press).

Nagel, Thomas (1971), "The Absurd", The Journal of Philosophy 68: 716-27.

- (1986), The View from Nowhere (New York, NY: Oxford University Press).

Parfit, Derek (1984), Reasons and Persons (New York, NY: Oxford University Press).

Núñez, Rafael, and Sweetser, Eve (2006), "With the Future Behind Them: Convergent Evidence From Aymara Language and Gesture in the Crosslinguistic Comparison of Spatial Construals of Time", Cognitive Science, 30: 401-50. 